\title{
FOLIA
}

Amazónica

Revista del Instituto de Investigaciones

de la Amazonía Peruana

\section{LOS ARCTIINI (LEPIDOPTERA, EREBIDAE, ARCTIINAE) ATRAÍDOS POR Heliotropium sp. (HELIOTROPIACEAE) EN LA ESTACIÓN BIOLÓGICA JOSÉ ÁLVAREZ ALONSO, IQUITOS, PERÚ}

\author{
Georgette Paola ANCAJIMA ${ }^{1,2, *}$, Diego Andrés NEYRA-HIDALGO ${ }^{3}$ \\ 1 Laboratório de Lepidoptera, Museu de Zoologia, Universidade de São Paulo. Ipiranga, São Paulo, \\ Brasil. \\ 2 Departamento de Entomología, Museo de Historia Natural, Universidad Nacional Mayor de San \\ Marcos - UNMSM. Lima, Perú. \\ 3 Facultad de Ciencias Biológicas. Universidad Nacional de la Amazonía Peruana - UNAP. Iquitos, \\ Perú. \\ * Correo electrónico: paolancajima@gmail.com
}

\section{RESUMEN}

Los árctinos son atraídos por plantas productoras de pirrolizidinas (ej. Heliotropiaceae), ya que emplean estos compuestos en las feromonas. La necesidad de este compuesto hace que las plantas secas de algunas Heliotropiaceae sean un eficiente método de colecta. Este estudio evaluó la riqueza de Arctiini con preferencia de Heliotropium sp. en la Estación Biológica José Álvarez Alonso, durante los meses de enero, febrero y marzo de 2014. Se establecieron 21 puntos de colecta, 14 puntos en bosque tropical y 7 en varillales. Fueron colectados 16 especies de Arctiini (39 ejemplares), distribuidos en las subtribus Arctiina, Ctenuchina y Euchromina. Los varillales mostraron mayor número de especies en relación a los bosques tropicales. Probablemente exista una preferencia por las hojas de Heliotropium sp. Todas las especies registradas son nuevos registros para la Estación Biológica José Álvarez Alonso, de los cuales, siete han sido exclusivamente registradas en los varillales. Se ha generado una base para futuros trabajos, es probable que estos bosques albergan un mayor número de especies, que podrían registrarse con mayor esfuerzo de muestreo y 
otros métodos de colecta. La lista presentada de la fauna de Arctiini sirve como soporte para la conservación de estos bosques.

PALABRAS CLAVE: árctinos, bosque tropical, varillales, pirrolizidina.

\title{
THE ARCTIINI (LEPIDOPTERA, EREBIDAE, ARCTIINAE) ATTRACTED BY Heliotropium sp. (HELIOTROPIACEAE) AT THE JOSÉ ÁLVAREZ ALONSO BIOLOGICAL STATION, IQUITOS, PERU
}

\begin{abstract}
Tiger moths are attracted to pyrrolizidine-producing plants (eg. Heliotropiaceae), since they use these compounds in pheromones. The need for this compound makes the dried plants of some borage plants an efficient collection method. This study evaluated the richness of Arctiini with preference of Heliotropium sp. at the José Álvarez Alonso Biological Station during the months of January, February and March 2014. Twenty-one sampling points were established, 14 points in tropical forest and 7 in varillales. 16 Arctiini species were collected (39 specimens), distributed in the Arctiina, Ctenuchina and Euchromina subtribes. The white-sand forest showed a greater number of species in relation to the tropical forests. There is probably a preference for the leaves of Heliotropium sp. All the registered species are new records for the José Álvarez Alonso Biological Station, of which seven have been exclusively registered in the white-sand forest. Additionally, a basis for future work has been generated, since with certainty, these forests host a greater number of species and that could be recorded with greater sampling effort and other collection methods. The presented list of the Arctiini fauna could serve as a support for the conservation of these forests.

KEYWORDS: tiger moths, tropical forest, white-sand forest, pyrrolizidine.
\end{abstract}




\section{INTRODUCCIÓN}

La Estación Biológica José Álvarez se encuentra ubicada en la Reserva Nacional Allpahuayo Mishana (RNAM) en la Amazonía occidental peruana. Esta reserva se encuentra en la Ecorregión Napo, conocida como una de las ecorregiones que concentra mayores niveles de biodiversidad en el mundo (Vargas et al., 2012), por lo que está considerada como alta prioridad para su conservación. La RNAM presenta gran riqueza de ecosistemas, incluyendo los bosques sobre arena blanca, localmente conocidos como varillales (García-Villacorta et al., 2003; Vargas et al., 2012). Los varillales tienen suelos pobres en nutrientes que permiten crecer vegetación monodominante (García-Villacorta et al., 2003), lo cual ha permitido la especialización de algunos animales (Anderson, 1981; Ruokolainen \& Tuomisto, 1993; Tuomisto, 1993), entre ellos, endémicos y muy poco estudiados (Álvarez et al., 2012). Entre los inventarios faunísticos realizados en la RNAM asociados a varillales se muestran registros de aves (Álvarez et al., 2012; Acosta-Diaz et al., 2019), anfibios, reptiles (Rivera et al., 2003; Acosta, 2016) y de algunos grupos de insectos (De la Cruz-Abarca et al., 2003; Campos \& Ramirez, 2005; Ampudia \& Estrella, 2020; Vásquez et al., 2021). Los trabajos sobre Lepidoptera en estas áreas son exiguos y se limitan a algunas familias de Papilionoidea (Campos \& Ramírez, 2005; Vásquez et al., 2021); esto refleja un déficit tanto de muestreos en áreas de importancia ecológica como de información sobre otros grupos de lepidópteros, ya que el orden contiene 43 superfamilias. Entre ellas, la superfamilia Noctuoidea es uno de los más numerosos en donde está incluida la subfamilia Arctiinae (Erebidae), la cual comprende 6000 especies en la región Neotropical (Watson \& Goodger, 1986), se estima la ocurrencia de 1600 especies en el Perú (Grados, 2011), se caracterizan por ser macrolepidópteros mayormente nocturnos, no obstante, existen especies crepusculares y diurnos (Scoble, 1995). Actualmente, la subfamilia está divida en cuatro tribus: Lithosiini, Amerilini, Syntomini y Arctiini (Zenker et al., 2016). De los cuales, Arctiini y Amerilini presentan especies con farmacofagia, es decir, ingieren compuestos químicos con fines no nutritivos, por ejemplo, los adultos consumen alcaloides pirrolizidínicos (PAs) para producir feromonas y mejorar el éxito del apareamiento (Pliske, 1975; Boppré, 1984, 1990; Zaspel et al., 2014). Estos compuestos químicos se encuentran en partes marchitas de algunas familias de plantas como Heliotropiaceae, Apocynaceae y Fabaceae (Bull et al., 1968), es por ello que el empleo de plantas secas de Heliotropium sp. (Heliotropiaceae) son eficaces atrayentes para la colecta de árctinos. Estas polillas pueden ser utilizados como indicadores de evaluaciones de diversidad (Grados et al., 2010; Grados et al., 2015; MINAM, 2018), debido a que son fáciles y económicos de muestrear, tienen especies con amplia distribución geográfica, cuentan con colecciones de referencia y existen especialistas trabajando con el grupo (Grados et al., 2010).

Este estudio evaluó la riqueza de Arctiini atraídos por Heliotropium sp. en los bosques de la Estación Biológica José Álvarez Alonso en la Reserva Nacional Allpahuayo Mishana.

\section{MATERIALES Y MÉTODOS}

Las evaluaciones se realizaron en la Estación Biológica José Álvarez Alonso del Instituto de Investigaciones de la Amazonía Peruana (IIAP) en la Reserva Nacional Allpahuayo (RNAM), en el distrito San Juan Bautista, Maynas - Loreto, durante los meses de enero, febrero y marzo 2014 (época de lluvias). Se establecieron 21 puntos de colecta en cuatro trochas pre-establecidas 
considerando dos tipos de vegetación: varillales (7 puntos) y bosque tropical (14 puntos) (Figura 1). En cada punto establecido se colgaron los atrayentes, plantas secas de Heliotropium sp., empleando la metodología de Lamas \& Pérez (1981), Grados (1999) y Grados et al. (2015)
(Figura 1). Los atrayentes fueron revisados cada dos horas entre las 08:00 a 22:00 horas de lunes a viernes, y se recorría una trocha por día.

Todos los ejemplares fueron sacrificados con cámaras letales, posteriormente colocados en sobres de papel glassine y trasladados al
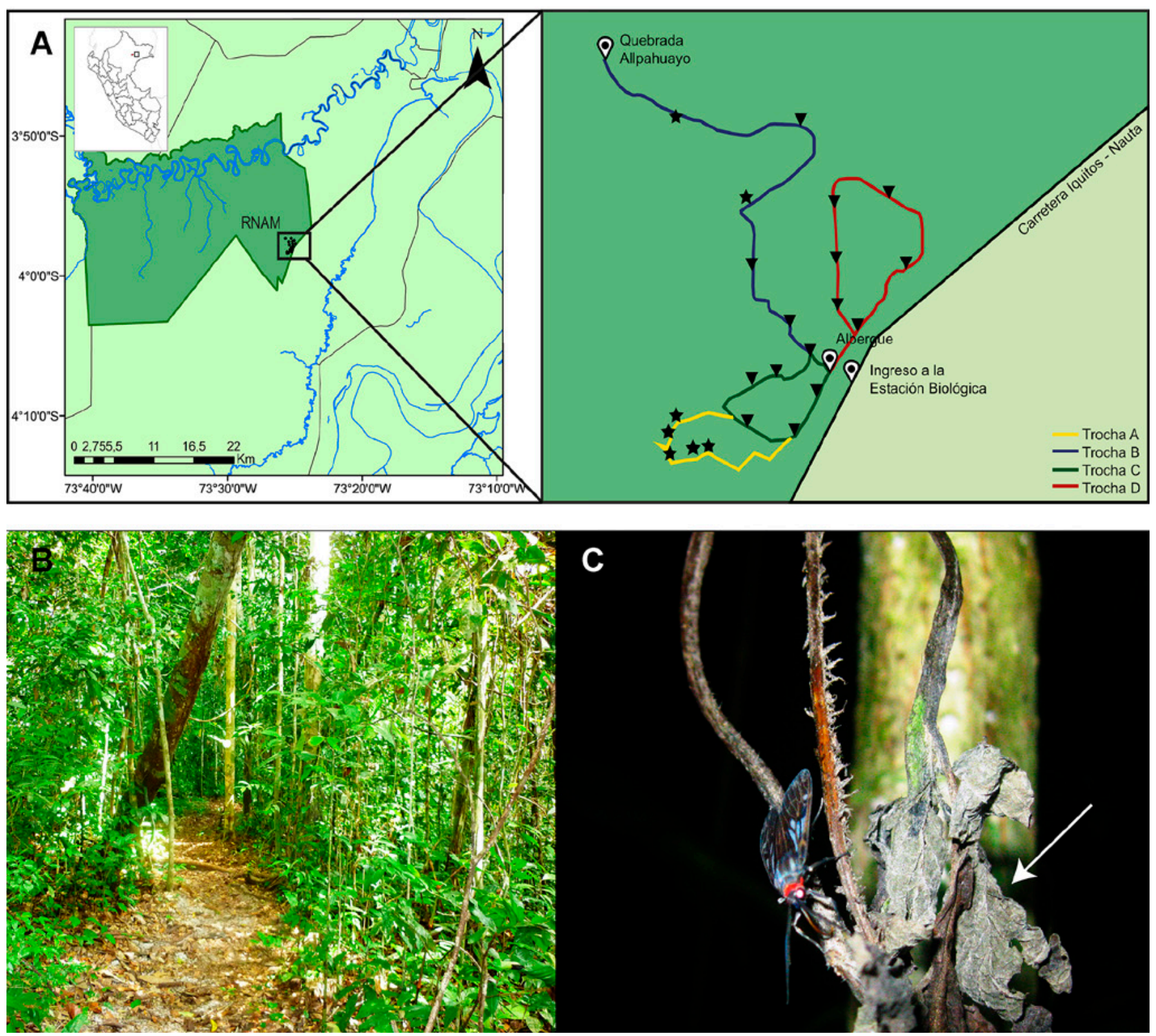

Figura 1: A. Ubicación del área de colecta y los puntos establecidos en la Estación Biológica José Álvarez Alonso (Reserva Nacional Allpahuayo Mishana) ( $\star$ : Varillales, $\mathbf{v}$ : Bosque tropical); B. Uno de los varillales donde se establecieron puntos de colecta; $\mathbf{C}$. Adulto de Hyaleucerea erythrotelus (Walker, 1854) alimentándose de las sustancias químicas de plantas secas de Heliotropium sp. (La flecha señala la planta seca de Heliotropium sp.). 
Tabla 1: Lista de especies de Arctiini registradas, sus abundancias, el tipo de vegetación y parte de la planta donde fueron capturadas. n: Número de individuos, Var: Varillal, Bos: Bosque tropical, Infloresc: Inflorescencia.

\begin{tabular}{|c|c|c|c|c|c|c|c|}
\hline \multirow[t]{2}{*}{ Subtribu } & \multirow[t]{2}{*}{ Especie } & \multirow[t]{2}{*}{$\mathrm{n}$} & \multicolumn{2}{|c|}{$\begin{array}{c}\text { Tipo de } \\
\text { vegetación }\end{array}$} & \multicolumn{3}{|c|}{$\begin{array}{l}\text { Parte de la planta donde } \\
\text { fueron capturados }\end{array}$} \\
\hline & & & Var. & Bos. & Raíz & Hojas & Infloresc. \\
\hline Arctiina & Pitane napravniki Grados, 2004 & 1 & & $x$ & & $x$ & \\
\hline \multirow{12}{*}{ Ctenuchina } & Aclytia klagesi (Rothschild, 1912) & 5 & $x$ & $x$ & & $x$ & $x$ \\
\hline & Cacostatia germana Rothschild, 1912 & 1 & & $x$ & & $x$ & \\
\hline & Centronia sp. & 1 & $x$ & & & $x$ & \\
\hline & Heliura sp. & 1 & $x$ & & & $x$ & \\
\hline & Hyaleucerea erythrotelus (Walker, 1854) & 7 & $x$ & $x$ & & $x$ & $x$ \\
\hline & Metastatia pyrrhorrhoea (Hübner, 1827) & 4 & $x$ & $x$ & & $x$ & $x$ \\
\hline & Pseudoaclytia opponens (Walker, 1864) & 4 & $x$ & $x$ & & $x$ & $x$ \\
\hline & Telioneura carmania (Druce, 1883) & 1 & $x$ & & & $x$ & \\
\hline & Timalus leucomelas (Walker, 1856) & 1 & $x$ & & & $x$ & \\
\hline & Tipulodes rubriceps Dognin, 1912 & 1 & $x$ & & & $x$ & \\
\hline & Uranophora leocotelus (Butler, 1876) & 2 & $x$ & & & $x$ & \\
\hline & Xanthopleura perspicua (Walker, 1856) & 2 & $x$ & $x$ & & $x$ & $x$ \\
\hline \multirow{3}{*}{ Euchromina } & Dixophlebia quadristrigata (Walker, 1864) & 4 & $x$ & & $x$ & & $x$ \\
\hline & Pleurosoma sp. & 2 & $X$ & $x$ & & $x$ & $x$ \\
\hline & Pseudopistosia sp. & 2 & $x$ & $x$ & & $x$ & \\
\hline
\end{tabular}

departamento de Entomología del Museo de Historia Natural, Universidad Nacional Mayor de San Marcos - Lima (Perú) (MUSM), donde se realizó el montaje, la identificación taxonómica y el depósito de todo el material colectado. Los ejemplares fueron identificados mediante la consulta de las descripciones originales y la revisión de material depositado en el MUSM.

\section{RESULTADOS Y DISCUSIÓN}

Se registraron 16 especies de Arctiini (Tabla 1). Los 39 ejemplares capturados corresponden a tres subtribus de Arctiini, 30 individuos de Ctenuchina (12 especies), 8 individuos de Euchromina (3 especies) y sólo un ejemplar de Arctiina (1 especie) (Tabla 1). La especie Hyaleucerea erythrotelus (Walker, 1854) fue la más abundante (Tabla 1). En relación a los tipos de vegetación evaluados, varillales registró 14 especies, siendo siete exclusivas de esta vegetación; mientras que en bosques tropicales se registraon nueve especies, siendo dos de ellas exclusivas de este tipo de vegetación. También se observó que siete especies pueden encontrarse en ambos tipos de vegetación: Aclytia klagesi (Rothschild, 1912), Hyaleucerea erythrotelus (Walker, 1854), Metastatia pyrrhorrhoea (Hübner, 1827), Pseudoaclytia opponens (Walker, 1864), Pleurosoma sp., Pseudopistosia sp. y Xanthopleura perspicua (Walker, 1856) (Tabla 1).

Se registró mayor número de especies posadas sobre las hojas (15) de Heliotropium sp. (Figura 
1C, Tabla 1, Figura 2), sin embargo, sólo ocho de ellas fueron capturadas exclusivamente sobre las hojas $(n=26)$. No se registró preferencias exclusivas en la raíz o la inflorescencia (Tabla 1 , Figura 2).

Esta investigación constituye un primer listado de Arctiini en los bosques de la Estación Biológica José Álvarez Alonso. El número de especies registrados es bajo en comparación con otras localidades como la Reserva Nacional Tambopata (RNTMB) (Madre de Dios) (199 especies) (Grados, 1999), Bajo Río Urubamba (Cusco) (241 especies) (Grados, 2001), Santuario Histórico de Machu Picchu (SHM) (Cusco) (80 especies) (Grados, 2002) y el Parque Nacional Bahuaja Sonene (PNBS) (Puno) (327 especies)
(Grados et al., 2015), esto se debe principalmente a que en estos sectores, las colectas fueron realizadas principalmente con trampas de luz y complementadas con atrayentes de Heliotropium sp. (Grados, 1999, 2001, 2002; Grados et al., 2015). Mientras que, en nuestro caso, solo reportamos los árctinos atraídos por plantas de Heliotropium sp.

La homogeneidad de vegetación y los suelos pobres en nutrientes de los varillales trae como consecuencia alto niveles de endemismo, poca riqueza y dominancia de pocas especies (Anderson, 1981; Rivera et al., 2003). Nuestro estudio mostró que los varillales tuvieron mayor número de especies de árctinos en comparación con los bosques tropicales, pese

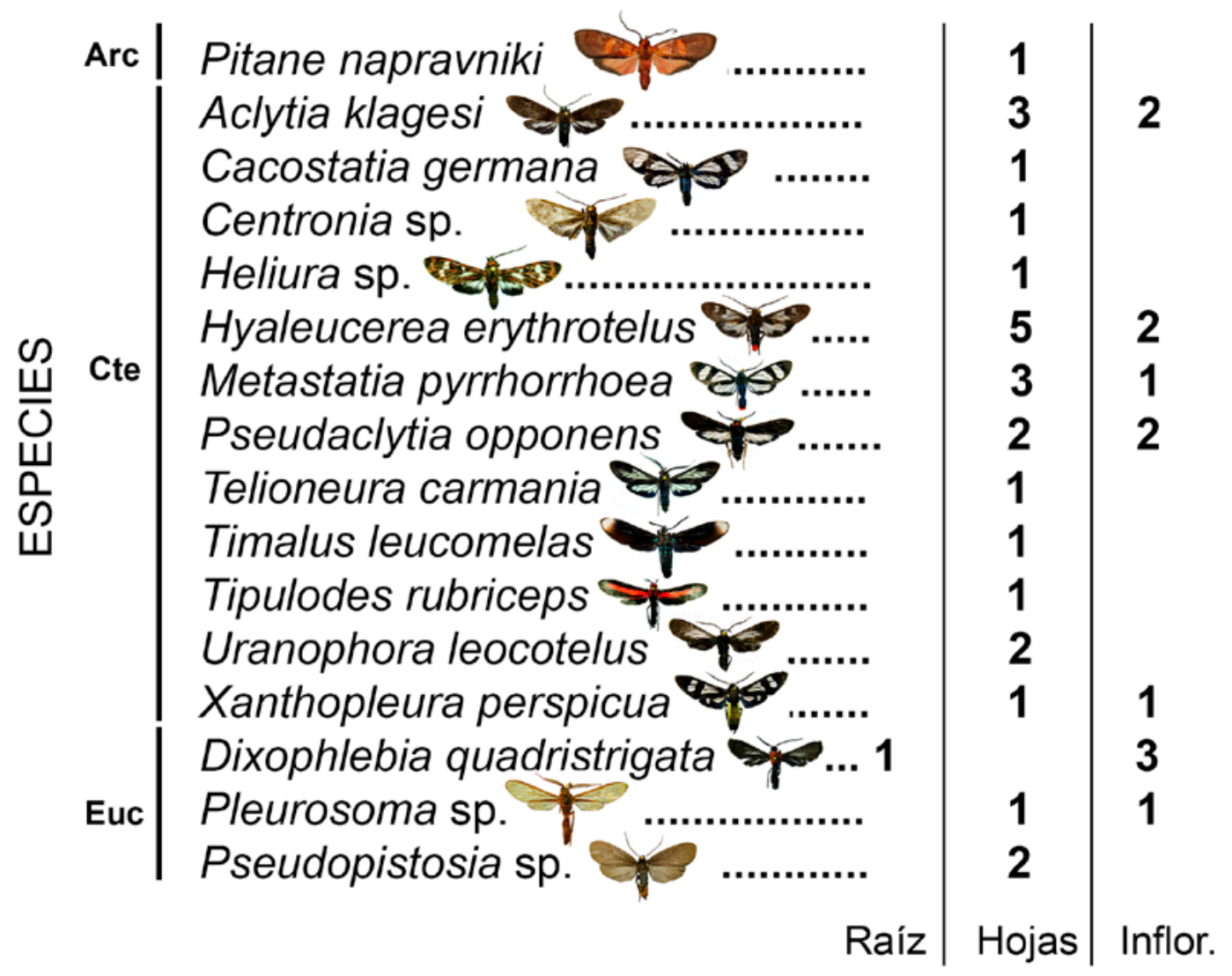

Figura 2: Abundancias registradas según parte de la planta Heliotropium sp. donde se capturaron las especies. Arc: Arctiini; Cte: Ctenuchini; Euc: Euchromini; Inflor.: Inflorescencia. 
a que en varillales se tuvo menos puntos de colecta. Nuestro resultado contrasta con los estudios antes mencionados que indican una menor riqueza de especies en varillales, sin embargo, como se registra en nuestro estudio, la partición de los recursos alimenticios (por ejemplo: el uso de diferentes partes de la planta atrayente) podría promover una mayor riqueza de especies en este hábitat. De las especies que sólo fueron registradas en varillales, Telioneura carmania, Tipulodes rubriceps y Dixophlebia quadristrigata también han sido registradas en bosques tropicales de la RNTMB y en Bajo Río Urubamba (Grados, 1999, 2001). Estas tres especies fueron registradas en bosques secundarios y en vegetación con dominancia de bambú (Grados, 2001), lo cual podría sugerir que son especies que prefieren hábitats perturbados u homogéneos.

El uso de plantas de Heliotropium como atrayente de polillas y mariposas ha sido empleado en varias investigaciones (Lamas \& Pérez, 1981, Grados, 1999, Grados et al., 2015), sin embargo, no se ha detallado en que parte de la planta se han encontrado alimentándose las especies atraídas. Nuestros resultados muestran una posible preferencia por las hojas, hecho que podría estar correlacionado con una mayor acumulación de alcaloides de pirrolizidinas (PAs) en esta región, o con la partición de este recurso por parte de los árctinos (Wisheu, 1998).

En conclusión, este trabajo revela nuevos registros de Arctiini para la Estación Biológica José Álvarez Alonso, de los cuales, siete han sido exclusivamente registradas en los varillales, bosques únicos de la Amazonía. Se presume que este tipo de bosque podría albergar una alta riqueza de especies de lepidópteros, sin embargo, se requiere emplear mayor esfuerzo de muestreo y aplicar otros métodos de colecta. Se recomienda ampliar el muestreo en la zona estudiada abarcando los diferentes tipos de bosque de la estación,así mismo utilizando trampas de luz y redes entomológicas para capturar los Arctiini que no son atraídos por plantas secas de Heliotropium sp. Por último, pese a que nuestro estudio se llevó a cabo dentro de un área protegida por el estado peruano, existen amenazas que pondrían en riesgo la fauna local, es por ello que este tipo de inventarios rápidos son importantes para generar soportes para adoptar medidas de conservación adecuadas.

\section{AGRADECIMIENTOS}

A Freddy Arévalo por el soporte durante mi estadía en la Estación Biológica y proporcionarme los shapefiles para la elaboración del mapa. Al Blgo. Juan Grados por la confirmación las identificaciones de las especies. A Francisco Vásquez y Nancy Arévalo por el alojamiento durante todo el tiempo de colecta. A Wellington Ferreira y Mabel Alvarado por todos los alcances en la escrita del manuscrito.

\section{REFERENCIAS BIBLIOGRÁFICAS}

Acosta-Diaz, A.; Ayapi-Silva, J.; OcampoRodríguez, M.; Gálvez H. 2019. Riqueza y características morfométricas de aves de sotobosque en Varillal Alto Seco de la Reserva Nacional Allpahuayo Mishana, Perú. Revista de Investigaciones Veterinarias del Perú, 30(2): 709-720. DOI: http://dx.doi.org/10.15381/ rivep.v30i2.16074

Acosta, A. 2016. Bioecologia de Dendrobates reticulatus Boulenger, 1883 (Anura: Dendrobatidae) en Varillal Alto Seco de la Reserva Nacional Allpahuayo - Mishana, Iquitos. Tesis de doctorado, Universidad Nacional Federico Villarreal, Escuela Universitaria De Post Grado, Lima, Perú. 207pp. 
Álvarez, J.; Díaz J.; Shany, N. 2012. Avifauna of the Allpahuayo-Mishana National Reserve, Loreto, Peru. Cotinga, 34(1): 132-152.

Ampudia, C.; Estrella, R. 2020. Efectividad de cebos en la captura de escarabajos saprófagos (Insecta: Coleoptera) en Allpahuayo Mishana, Amazonía peruana. Ciencia amazónica (Iquitos), 8(1): 71-84. DOI: http://dx.doi. org/10.22386/ca.v8i1.281

Anderson, A. 1981. White sand vegetation of Brazilian Amazonia. Biotropical, 13(3): 199210. DOI: https://doi.org/10.2307/23881255

Boppré, M. 1984. Redefining "Pharmacophagy". Journal of Chemical Ecology, 10(7): 11511154.

Boppré, M. 1990. Lepidoptera and pyrrolizidine alkaloids - Exemplification of Complexity in Chemical Ecology. Journal of Chemical Ecology, 16(1): 165-185.

Bull, L; Culvenor, C.; Dick, T. 1968. The pyrrolizidine Alkaloids. Amsterdam, North Holland. 293 pp.

Campos, L.; Ramírez, J. 2005. Diversidad, Patrones de Distribución y Estructura de Comunidades de las Mariposas de la Zona Reservada AllpahuayoMishana, Loreto, Perú. Tesis de pre-grado, Universidad Nacional de la Amazonía Peruana, Facultad de Ciencias Biológicas, Loreto, Perú. $90 \mathrm{pp}$.

De la Cruz, A.; Aibar, P.; Campos, L.; Polo, R.; Ríos, S.; Valencia, G.; Santisteban, J. 2003. Evaluación de Hymenoptera y Coleoptera (Insecta) en diferentes tipos de vegetación de la Zona Reservada Allpahuayo - Mishana, Loreto, Perú. Folia Amazónica, 14(1):133-138. DOI: $10.24841 /$ fa.v14i1.160

García-Villacorta, R.; Ahuite-Reátegui, M.; Olórtegui-Zuameta, M. 2003. Clasificación de Bosques sobre arena blanca de la Zona Reservada Allpahuayo-Mishana. Folia Amazónica, 14(1): 17-33. DOI: 10.24841/fa.v14i1. 151
Grados, J. 1999. Lista Preliminar de los Ctenuchinae (Lepidoptera: Arctiidae) de la Zona Reservada Tambopata-Candamo. Revista Peruana de Entomología, 41: 9-14.

Grados, J. 2001. Lista de los Ctenuchinae (Lepidoptera:Arctiidae) de la región del Bajo Urubamba, Cuzco, Perú. Revista Peruana de Entomología, 42: 61-67.

Grados, J. 2002. Los Arctiidae y Sphingidae (Lepidoptera: Heterocera) del Santuario Histórico de Machu Picchu, Cuzco, Perú: Estudio Preliminar. Revista peruana de biología, 9(1): 16-22. DOI: https://doi.org/10.15381/ rpb.v9i1.2516.

Grados, J.; Figueroa, L.; Alvarado, M. 2010. Grupos Indicadores y Representativos de Insectos en evaluaciones rápidas de diversidad $y$ evaluaciones de impacto ambiental. Libro de resúmenes de LII Convención Nacional de Entomología.

Grados, J. 2011. Los artrópodos de la Mancomunidad Saywite-Choquequirao-Ampay (Apurímac, Perú). En: Baiker, J. (Ed.). Guía ecoturística: Mancomunidad SaywiteChoquequirao-Ampay (Apurímac, Perú). Con especial referencia a la identificación de fauna, flora, hongos y líquenes en el departamento de Apurímac y sitios adyacentes en el departamento de Cusco. Serie Investigación y Sistematización $\mathrm{N}^{\circ}$ 15. Programa Regional ECOBONA - INTERCOOPERATION, Lima. 968 pp.

Grados, J.; Baynes, H.; Rázuri, E.; Figueroa, L.; Barrientos, J.; Guillermo, E. 2015. Insectos. Invisiblemente mágico. In Montoya, M.; Cossios, D.; Silva, M.; Coll, D. (Eds.). Parque Nacional Bahuaja Sonene. Inventarios Biológicos Rápidos (Segunda Edición), Wildlife Conservation Society. p. 92-107.

Lamas, G.; Pérez, E. 1981. Danainae e Ithomiinae (Lepidoptera, Nymphalidae) atraídos por 
Heliotropium (Boraginaceae) en Madre de Dios, Perú. Revista Peruana de Entomología, 24 (1): 59-62.

Ministerio del Ambiente, 2018. Guía para la elaboración de la Línea Base en el Marco del Sistema Nacional de Evaluación del Impacto Ambiental, Lima, Perú. 227pp.

Pliske, T. 1975. Attraction of Lepidoptera to plants containing pyrrolizidine alkaloids. Environmental Entomology, 4(3): 455-479. DOI: https://doi.org/10.1093/ee/4.3.455

Rivera, C.; vonMay, R.; Aguilar, C.; Arsita, I.; Curo, A.; Schulte, R. 2003. Una evaluación preliminar de la herpetofauna en la Zona Reservada Allpahuayo - Mishana, Loreto, Perú. Acta Amazónica, 14(1-2): 138-148. DOI: 10.24841/ fa.v14i1.161

Ruokolainen, K.; Tuomisto H. 1993. La vegetación de terrenos no inundables (tierra firme) en la Selva Baja de la Amazonía Peruana. En: Kalliola, R.; Puhakka, M.; Danjoy, W. (Eds). Amazonía Peruana, Vegetación húmeda tropical en el llano subandino. p. 139-153.

Scoble, M. 1995. The Lepidoptera: form, function, and diversity. Oxford University Press, Oxford, 404pp.

Vargas, V.; Stauffer, F.; Pintaud, J. 2012. Riqueza, Usos y Conservación de palmas (Arecaceae) en la Reserva Nacional Allpahuayo Mishana (Perú). Acta Botánica Venezuelica, 35(1): 5370.
Vásquez, J.; Callirgos, J.; Zárate, R.; RamírezHernandez, J.; Pinedo, J., García, A.; Valderrama, H.; Pacheco, T.; Tello, R. 2021. Diversidad y composición de mariposas (Lepidoptera: Morphinae y Satyrinae) de los varillales en la Reserva Nacional Allpahuayo Mishana, Loreto, Perú. Boletín Científico. Centro de Museos, 25(1), 177-190. DOI: https://doi. org/10.17151/bccm.2021.25.1.11

Watson, A.; Goodger D.T. 1986. Catalogue of the Neotropical Tiger-moths. Occasional Papers on Systematics Entomology, 1: 1-71.

Wisheu, I. 1998. How organisms partition habitats: different types of community organization can produce identical patterns. OIKOS, 83(2): 246-258. DOI: https://doi. org/10.2307/3546836.

Zaspel, J.M.; Weller, S.J.; Wardwell, C.T.; Zahiri, R.; Wahlberg, N. 2014 Phylogeny and Evolution of Pharmacophagy in Tiger Moths (Lepidoptera: Erebidae: Arctiinae). PLoS ONE, 9(7): e101975. DOI: https://doi.org/10.1371/journal.pone. 0101975.

Zenker, M.M.; Wahlberg, N.; Brehm, G.; Teston, J.A.; Przybylowicz, L.; Pie, M.R.; Freitas, A.V.L. 2016. Systematics and origin of moths in the subfamily Arctiinae (Lepidoptera, Erebidae) in the Neotropical region.Zoologica Scripta, 1-15. DOI: https://doi.org/10.1111/zsc.12202.

Recibido: 22 de marzo de 2021 Aceptado para publicación: 4 de junio de 2021 